\title{
Beauty photoproduction at ZEUS
}

\author{
Volodymyr Aushev ${ }^{\mathbf{1}}$ \\ (on behalf of the ZEUS Collaboration)
}

DESY/INR

Notkestrasse 85, Hamburg/ Germany D-22603

E-mail: volodymyr.aushev@desy.de

Photoproduction of beauty quarks in events with two jets has been measured with the ZEUS detector at HERA using an integrated luminosity of $128 \mathrm{pb}^{-1}$. The beauty content has been extracted using the decay length significance of the $\mathrm{B}$ hadrons and the invariant mass of the decay vertices. Differential cross sections as a function of $P_{t}^{\text {jet }}$ and $\eta^{\text {jet }}$ are measured and compared with the PYTHIA leading order predictions. The differential cross-section as a function of $\eta^{\text {jet }}$ is compared to a previously published HERA I results.

XVIII International Workshop on Deep-Inelastic Scattering and Related Subjects - DIS 2010

Firenze, Italy

April 19-232010

\footnotetext{
$1 \quad$ Speaker
} 


\section{Introduction}

The study of beauty production remains a very interesting topic in high energy physics since it constitutes a rigorous test of perturbative quantum chromodynamics (pQCD). One of the main ingredients in the measurement of beauty quark cross sections is the determination of the beauty fraction in the relevant data sample. Different experimental techniques have been developed to discriminate beauty from charm and light flavour background. These procedures rely often on characteristic features of the beauty production, mostly related to its heavy mass or long lifetime compared to lighter quarks. All these experimental procedures used so far in ZEUS to measure beauty production are limited to the semileptonic beauty decays [1]. The work presented in this report deals with an inclusive beauty measurement in which the hadronic decays are also taken into account. For that purpose, a new method for beauty identification based on the reconstruction of the decay vertices (secondary vertices) has been developed. Due to the relatively long lifetime of $\mathrm{b}$ hadrons, beauty secondary vertices are considerably displaced with respect to the interaction vertex compared to the decay vertices of charm and light quarks. This fact will be exploited by means of a signed transverse decay length together with the distribution of the invariant mass of the secondary vertices to statistically extract the fraction of beauty in the sample. Beauty cross sections and differential cross sections $d \sigma / d p_{T}$ and $d \sigma / d \eta$ are then measured in the photoproduction regime. The obtained results are compared to previous measurements and to theoretical predictions from NLO QCD calculations.

\section{Event selection and signal extraction}

A sample of photoproduction events (with photon virtuality $Q^{2}<1 \mathrm{GeV}^{2}$ ) with two jets was selected from $e^{+} p$ collisions, corresponding to an integrated luminosity of $128 \mathrm{pb}^{-1}$. The energies of the proton and positron beams were $920 \mathrm{GeV}$ and $27.6 \mathrm{GeV}$, respectively, corresponding to a centre-of-mass energy of $\sqrt{s}_{s}=318 \mathrm{GeV}$. Jets were selected if they fulfilled the requirements $E_{T}^{j e t}>7(6) G e V$ and $\left|\eta^{j e t}\right|<2.5$. In order to reconstruct the B hadron decay vertices, tracks have been selected which had a transverse momentum of $p_{t} \geq 0.5 \mathrm{GeV}$ and were well-reconstructed in the central tracking detector (CTD) and the micro-vertex detector (MVD).

The heavy flavour content of the selected sample was determined by means of the decay length significance, $S$, which is defined as the decay length $d$, projected on the $x-y$-plane and subsequently projected onto the jet-axis, divided by its error $\delta d$. It is combined with the invariant mass, $\mathrm{m}_{\mathrm{vtx}}$, of the secondary vertex tracks.
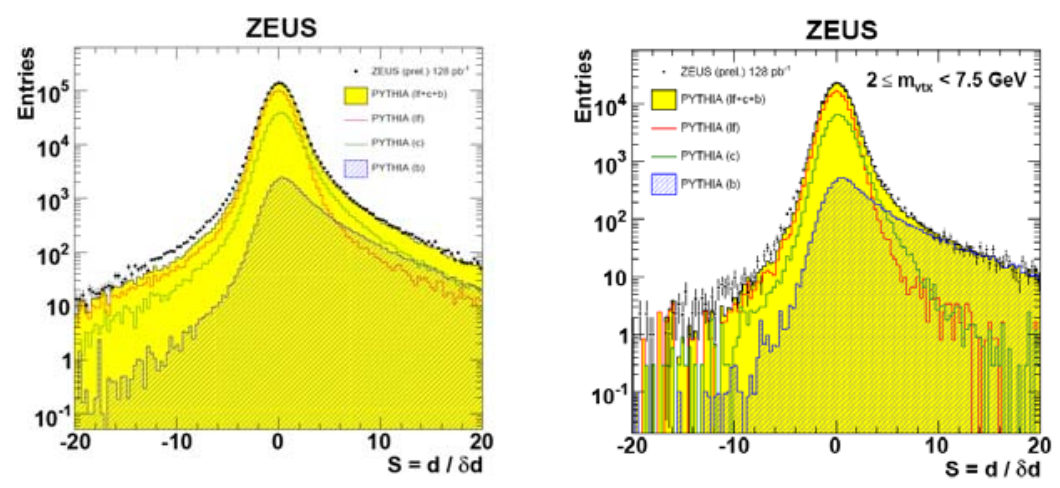

Fig.1. Distribution of the decay length significance of selected secondary vertices for the total range (left) and in the region $2<m_{v t x}<7.5 \mathrm{GeV}$ (right). The data are compared to the sum of all Monte Carlo distributions and to the individual contributions. The Monte Carlo was scaled using the result of the fit. 
The decay length significance distribution was divided into different mass bins in order to provide an almost pure beauty region at $2 \mathrm{GeV}<\mathrm{m}_{\mathrm{vtx}}<7.5 \mathrm{GeV}$, while the lower mass bins are dominated by charm. Fig.1 shows $S$ for the total and the highest mvtx range.

The left side of the significance $\left(S^{-}, S<0\right)$ is mirrored onto and subtracted from the right side $\left(S^{+}, S>0\right)$ in order to reduce the related systematic effects. In order to extract the contributions from $b, c$ and lf in the sample a binned $\chi^{2}$ fit of the mirrored significance distributions $\left(S+-S^{-}\right)$simultaneously in all three mass bins was performed. The overall MC normalisation was constrained by adjusting the relative normalisation of the three MC subsamples to be consistent with the normalisation of the data in the significance distribution. Fig.2 shows $S^{+}-S^{-}$in three mass bins after the fit. The same fit has been done in differential bins in $P_{\mathrm{T}}^{\text {Jet }}$ and $\eta^{\text {Jet }}$.

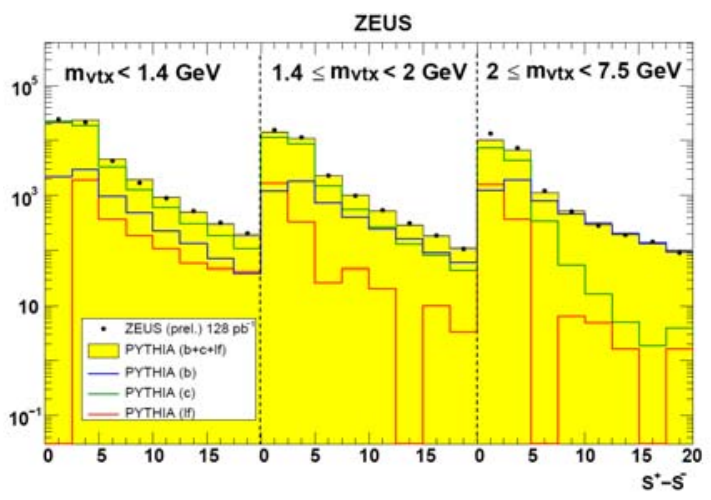

Fig.2. Distribution of the mirrored decay length significance in three mass bins, displaying the data and total MC distributions as well as the contributions from the three MC sub-samples normalised according to the fractions obtained from the fit.

Requiring $m_{v t x}>2 \mathrm{GeV}$ and $S^{+}-S^{-}>8$ it was possible to obtain an almost pure beauty sample, for which good agreement between data and MC was found (Fig. 3).
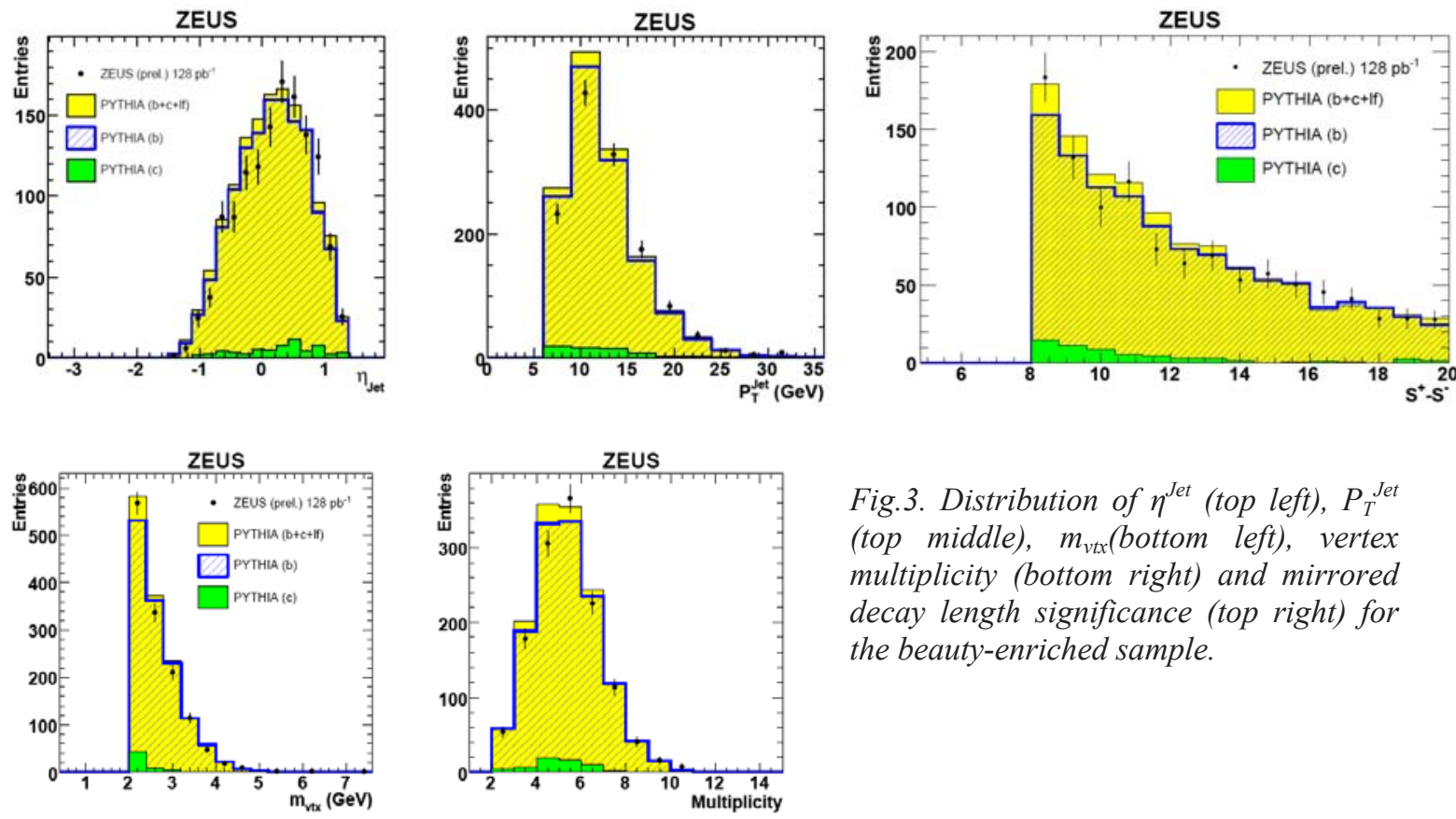

Fig.3. Distribution of $\eta^{\text {Jet }}$ (top left), $P_{T}^{\text {Jet }}$ (top middle), $m_{v t x}$ (bottom left), vertex multiplicity (bottom right) and mirrored decay length significance (top right) for the beauty-enriched sample. 


\section{Results}

The differential cross-sections as a function of $P_{T}^{\text {Jet }}$ and $\eta^{\text {Jet }}$ were measured for the process $e+p \rightarrow e+b^{-} b X$ in the kinematic range $Q^{2}<1 \mathrm{GeV}^{2}, 0.2<y<0.8, P_{T}^{\text {Jetl (2) }}>7(6) \mathrm{GeV}$, $-2.5 \leq \eta^{\text {Jet } 1(2)}<2.5$ with at least one of the jets within $-1.6<\eta^{\text {Jet } 1(2)}<1.3$. For the acceptance $\mathrm{B}$ hadron jets were calculated using the final state particles before the decay of the weakly decaying B hadron. The results are compared to the Pythia LO prediction as well as an NLO QCD prediction calculated using the FMNR programme[3] as shown in Fig. 4. The FMNR settings are the same as in [2] with the default scale $\mu=\mu_{0} / 2$. Good agreement between the measurement and the predictions was observed.
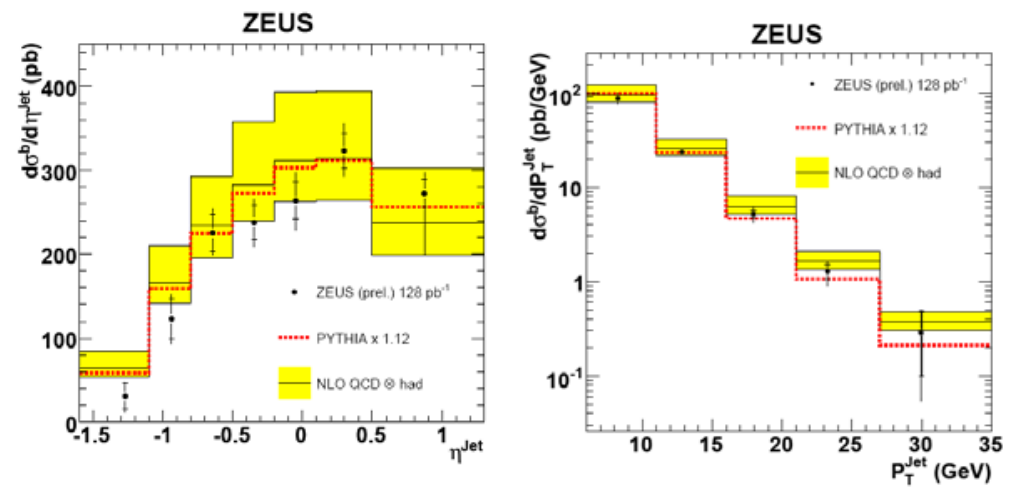

Fig.4. Differential cross section as a function of $P_{T}^{\text {Jet }}$ (right) and $\eta^{\text {Jet }}($ left). The black points show the results from this analysis. The inner error bars are statistical uncertainties, while the external error bars show the statistical and systematic uncertainties. The band represents the NLO QCD prediction with uncertainties.

The cross-sections as a function of $\eta^{J e t}$ are compared to a previously published HERA I analysis [1] (Fig. 5). Both measurements agree well within the statistical and systematic errors. The errors on the measurement could be reduced substantially compared to the previous analysis. The main contributions to the systematic uncertainty are the description of the tails of the decay-length distribution which was estimated by smearing the $\mathrm{MC}$ to reproduce the data and the uncertainty in the trigger efficiency. Other contributions include a variation of the fit range, the hadronic energy scale uncertainty and uncertainties in the luminosity measurement.

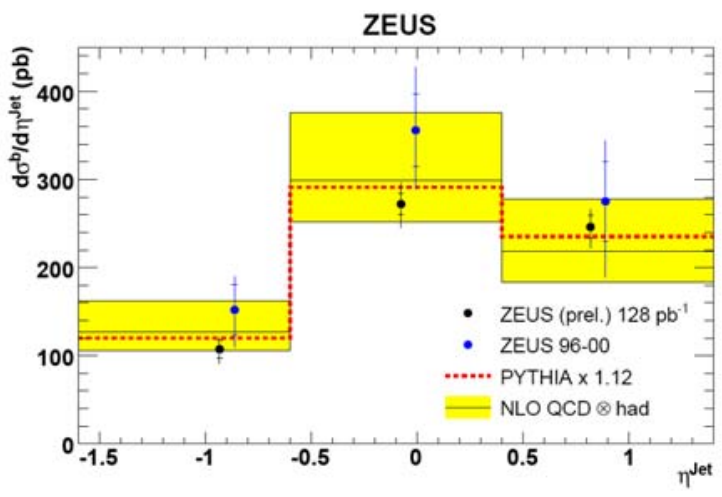

Fig.5. Differential cross-section as a function of $\eta^{\text {Jet }}$. The black points show the results from this analysis, the blue ones the results from the previously published HERA-I measurement. The inner error bars are statistical uncertainties, while the external error bars show the statistical and systematic uncertainties. The band represents the NLO $Q C D$ prediction and the theoretical uncertainties.

In Fig.6 a summary of the differential cross-sections for $b$-quark production as a function of $p_{t}^{b}$ measured by the ZEUS and H1 collaborations is presented. This measurement is in good agreement with all previous analyses shown. 


\section{HERA}

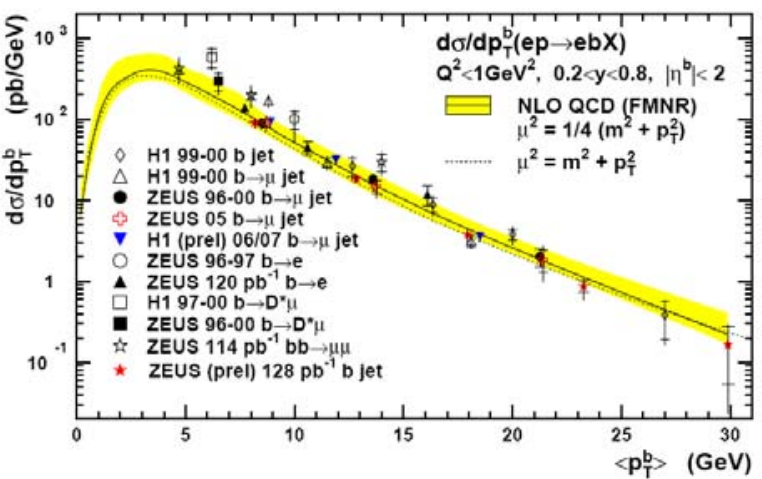

Fig.6. Summary of differential cross-sections for b-quark production as function of $p_{t}^{b}$ as measured by the ZEUS and H1 collaborations. The measurements are shown as points, with the result of this measurement shown as red stars. The inner error bars are the statistical errors; the outer bars show the statistical and systematic errors. The band represents the NLO QCD prediction and the theoretical uncertainties.

\section{Conclusion}

A measurement of beauty photoproduction from HERA II $e^{+} p$ - data has been presented. The decay length significance, $S$, and the invariant mass, $m_{v t x}$, of the B hadron decay vertex have been used to determine the heavy quark contributions to the selected dijet sample. The production cross-sections were found to be compatible with previous ZEUS and H1 measurements and with NLO QCD predictions, while significantly increasing the precision of the measurement.

\section{References}

[1] S. Chekanov et al., Beauty photoproduction measured using decays into muons in dijet events in ep collisions at $\sqrt{S}_{\mathbf{s}}=318 \mathrm{GeV}$, Phys. Rev D70:012008, 2004

[2] ZEUS Collaboration; S. Chekanov et al., Preprint DESY-08-210 (December 2008), 2008.

[3] S. Frixione et al., Phys. Lett. B 348, 633 (1995). 